\title{
Comparative Study of Plantar Pressure During Step Exercise in Different Floor Conditions
}

\author{
Rita Santos-Rocha ${ }^{1,2}$ and António Veloso ${ }^{2}$ \\ ${ }^{1}$ Technical University of Lisbon and ${ }^{2}$ Polytechnic Institute of Santarém
}

\begin{abstract}
Mechanical load has been estimated during step exercise based on ground reaction force (GRF) obtained by force platforms. It is not yet accurately known whether these measures reflect foot contact forces once the latter depend on footwear and are potentially modified by the compliant properties of the step bench. The aim of the study was to compare maximal and mean plantar pressure (PP), and maximal GRF obtained by pressure insoles after performing seven movements both over two metal force platforms and over the step bench. Fifteen stepexperienced females performed the movements at the cadences of 130 and 140 beats per minute. PP and GRF (estimated from PP) obtained for each floor condition were compared. Maximal PP ranged from $29.27 \pm 9.94$ to $47.07 \pm 12.88$ $\mathrm{N} / \mathrm{cm}^{2}$ as for metal platforms, and from 28.20 \pm 9.32 to $43.00 \pm 13.80 \mathrm{~N} / \mathrm{cm}^{2}$ as for the step bench. Mean PP ranged from $11.09 \pm 1.62$ to $14.32 \pm 2.06 \mathrm{~N} / \mathrm{cm}^{2}$ (platforms) and from 10.71 \pm 1.54 to $14.22 \pm 1.77 \mathrm{~N} / \mathrm{cm}^{2}$ (step bench). GRF (normalized body weight) ranged from $1.43 \pm$ 0.14 to $2.41 \pm 0.24 \mathrm{BW}$ (platforms) and from $1.38 \pm 0.14$ to $2.36 \pm 0.19 \mathrm{BW}$ (step bench). No significant statistical differences were obtained for most of the comparisons between the two
\end{abstract}

Santos-Rocha is with the Laboratory of Biomechanics, Faculty of Human Movement, Technical University of Lisbon, and the Sport Sciences School of Rio Maior, Polytechnic Institute of Santarém, Portugal, and Veloso is with the Sport Sciences School of Rio Maior, Polytechnic Institute of Santarém, Portugal. conditions tested. The results suggest that metal force platform surfaces are suitable to assess mechanical load during this physical activity. The forces applied to the foot are similar to the softer step bench and the hard force platform surface. This may reflect the ability of the performers to adapt their movement patterns to normalize the impact forces in different floor conditions.

Key Words: biomechanical load, ecological validation

An important goal of sports biomechanics is the assessment of the mechanical load imposed on the body during competitive or recreational physical activities. In this sense, the measure of ground reaction force (GRF) is quite common in the biomechanical approach to the effects that recreational sports activities have on the musculoskeletal system (Farrington \& Dyson, 1995; Maybury \& Waterfield, 1997; Scharff-Olson et al., 1997; Bartlett, 1999; Santos-Rocha et al., 2002), being an essential parameter for biomechanical modeling and loading assessment (Nigg \& Herzog, 1999; Winter, 2004). Foot GRF is affected by the mechanical properties of three bodies: the foot, the footwear, and the ground and, in some cases, the sports equipment such as the step bench. Thus, it is important to determine whether differences in the mechanical characteristics in any of these factors influence the mechanical load applied on the musculoskeletal system. 
Nevertheless, GRFs occurring in sports performance and recreational activity are commonly obtained using force platforms in a laboratory environment, despite the fact that sports facilities where those activities occur are built using materials with substantially different mechanical properties than those used to build force platforms. Few studies have addressed these concerns (Dixon et al., 2000; Williams, 2000; Dyson \& Janaway, 2002). In the case of running kinematics, it is becoming clear that the use of treadmills may lead to inadequate conclusions regarding ground running (Williams, 2000). For example, Dixon et al. (2000) report that subjects reveal similar impact forces when running on surfaces with various levels of rigidity revealing an ability to adjust the limb kinematics to prevent excessive loading.

Stepexercise is an indoor recreational activity characterized by mild-to-moderate levels of exertion and impact forces above those sustained during walking and similar to those registered during jogging or high-impact aerobics (Farrington \& Dyson, 1995; Maybury \& Waterfield, 1997; Scharff-Olson et al., 1997; Santos-Rocha et al., 2002). The stepping rate is usually set by music and varies from 120 to 150 beats per minute (bpm), corresponding to 30 to 37.5 cycles per minute. Usually each movement consists of two phases of loading for each leg stepping on (ascending phase) and stepping off (descending phase) of the step bench. Foot contact during the ascending phase is made to a surface with dampening properties (the step bench), whereas the contact during the descending phase is usually made with a sports facility surface (usually wood). The findings of Salem et al. (2000) suggested that despite step bench structural rigidity differences, plantar foot reaction forces during exercise remain similar, suggesting a mechanism regulating lower extremity stiffness and plantar foot loading.

One characteristic of a step session is the repetition of movements that produce low magnitude GRFs (around 1-2 BW) and of high frequency (around 3,900-4,200 loading phases during a 30min session, using music speed at 130-140 bpm). Accurate measures of GRF are essential to inform us about mechanical load during sport practices and to develop biomechanical models. During step exercise, mechanical load has been estimated based on GRF obtained by force platforms but it remains to be known how accurately these measures reflect foot contact forces because the latter depend on footwear and are potentially modified by the compliant properties of the step bench.

In-shoe plantar pressure measurement systems are capable of measuring pressures at the interface between the shoe and the foot. This equipment can be used in clinical, rehabilitation, and sports fields in order to assess patterns of forces between the agent and the ground (Hughes et al., 1991; Santos et al., 2001; Ribeiro \& Mota, 2004). Compared with force platforms, pressure insoles have the advantage of measuring the PP directly applied to the foot during less constrained tasks and may be used to assess other parameters like force distribution along the plantar region or between right and left foot, including outside the laboratory, with relatively ease. Pressure insoles also allow calculation of the maximal vertical GRF (VGRF).

Having a surface usually constructed of metal, the structural rigidity of a force platform is substantially different from those materials used in step benches (Reebok University Press, 1994). Having in mind the usual conditions on which participants of recreational exercise programs interact with ground and equipment, it was hypothesized that there would be a significant difference between the magnitude of forces when seven common movements are performed on a step bench and over a force platform. Therefore, using pressure insoles (Novel Pedar, Münich, Germany) placed inside usual sports footwear, the purpose of the present study was to compare the maximal and mean PP and maximal VGRF during step exercise performed in different floor conditions.

\section{Methods}

Fifteen step-experienced female subjects $(M \pm S D$ age, $25.9 \pm 5.3$ years; body mass, $61.1 \pm 6.3 \mathrm{~kg}$; height, $1.68 \pm 0.06 \mathrm{~m}$ ) with no history of lower limb complaints of either orthopedic or neurological origin volunteered to participate in the study after being fully informed about the aims and procedures. The study was approved by the review committee of Faculty of Human Movement of the Technical University of Lisbon. All subjects were right footdominant. Subjects performed 8 trials of each single lead movement (right-basic-step; left-basic-step; right-run-step; left-run-step) and 16 trials of each alternate lead movement (knee-lift-step; knee-hop- 
step; knee triple repeater) over two force platforms of metal surface with one raised to simulate the step bench, and in a gym room with a conventional wood floor and using the appropriate 15-cm-high step bench (Reebok). One of the platforms (Advanced Mechanical Technology, Watertown, MA) was used to substitute the step bench during stepping up. The other platform (Kistler AG, Winterthur, Switzerland) was used during stepping down (Figure 1). No arm movements were added, and movements were carried out at 130 and $140 \mathrm{bpm}$. In both floor conditions, PP was obtained by pressure insoles (Novel Pedar) placed inside the shoes. The same sports shoes were used in all trials. None of the subjects reported discomfort due to the pressure insoles or that their stepping movement was affected in any way by their use. The subjects were allowed to familiarize themselves with floor conditions and cadences before data collection. The cadence of 130 $\mathrm{bpm}$ is considered regular, whereas the cadence of $140 \mathrm{bpm}$ is considered fast.

The movements are described as follows: 1) right-basic-step: right foot step up, left foot step up, right foot step down, left foot step down. The right foot climbs to the bench, such as in climbing stairs, followed by the left foot. On the bench, the transition between both feet is similar to walking. Then the right foot descends backward to the floor, followed by the left foot. On the floor, the transition between both feet is similar to walking. The leading foot does not change; 2) left-basic-step: left foot step up, right foot step up, left foot step down, right foot step down. This pattern is symmetric to right-basic-step

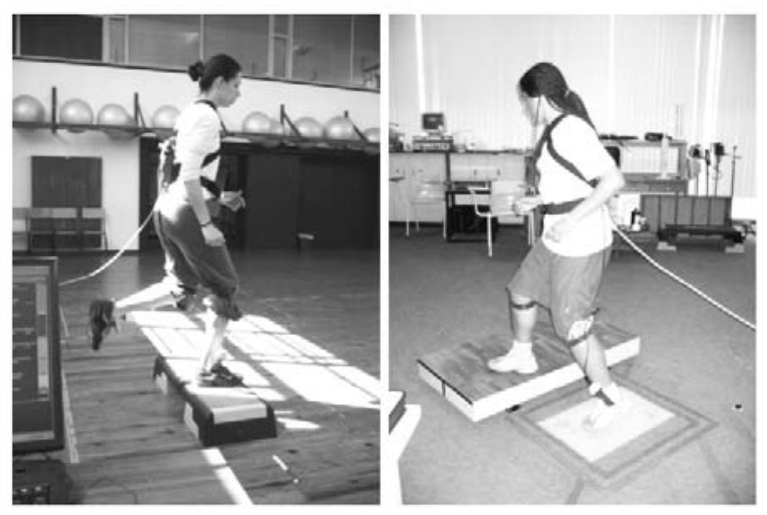

Figure 1 - Image of data collection using the pressure insoles when performing the movements over a step bench (on the left) and using the pressure insoles when performing the movements over two metal force platforms (on the right). because it starts with the left foot; 3) knee-lift-step: right foot step on, left knee lift, right foot step off, left foot step off, changing leading foot, left foot step on, right knee lift, left foot step off, right foot step off, changing leading foot. The right foot climbs to the bench, such as in climbing stairs, followed by the left foot lbblthat flexing the knee joint does a "knee lift." During this action the body is supported by the right limb on the bench. Then the left foot descends backward to the floor, followed by the right foot. On the floor the transition between both feet is similar to walking. Leading foot changes; 4) right-runstep: right foot step up with propulsion (leap), left foot step up with propulsion (leap), right foot step down, left foot step down. The right foot jumps to the bench followed by the left foot that also jumps to the bench. The transition between both feet is similar to running during ascending phase. The descending phase is similar to the right-basic-step: the right foot descends backward to the floor, followed by the left foot. On the floor the transition between both feet is similar to walking. Leading foot does not change; 5) left-run-step: left foot step up with propulsion (leap), right foot step up with propulsion (leap), left foot step down, right foot step down. This pattern is symmetric to right-run-step because it starts with the left foot; 6) knee-hop-step: right foot step on, left knee lift with propulsion (hop), left foot step off, right foot step off, changing leading foot, left foot step on, right knee lift with propulsion (hop), right foot step off, left foot step off, changing leading foot. The right foot climbs to the bench, such as climbing stairs, and jumps (hop), followed by the left foot lbblthat flexing the knee joint does a "knee lift" at the same time. The descending phase is similar to the right knee-lift-step: the left foot descends backward to the floor, followed by the right foot. On the floor the transition between both feet is similar to walking. Leading foot changes; 7) knee-triple-repeater: right foot step on, left knee lift three times, left foot step off, right foot step off, changing leading foot, left foot step on, right knee lift three times, right foot step off, left foot step off, changing leading foot. The right foot climbs to the bench such as climbing stairs, followed by the left foot lbblthat flexing the knee joint does three repetitions of a "triple knee lift". During this action the body is supported by the right limb on the bench. The descending phase is similar to the knee-lift-step and to the knee-hop-step: the left foot descends 
backward to the floor, followed by the right foot. On the floor the transition between both feet is similar to walking. Leading foot changes.

Plantar pressure signals were sampled at $50 \mathrm{~Hz}$, collected using Pedar-Online and processed using Pedar-Expert 8.3 software. Pedar-Analysis was used to visualize the original files. Hughes et al. (1991) indicate that pressure data from at least three to five steps are required if data are to be replicable. In the present study, for each subject, five trials of each single lead step (right or left) and 10 trials of each alternate lead step (right and left), on each condition, were selected for analysis using Pedar-Filter. The software calculates VGRF by summing the maximal PP of each of the 99 cells and dividing by the area of the foot. Pedar-Online was used to obtain maximal and mean PP $\left(\mathrm{N} / \mathrm{cm}^{2}\right)$ and maximal VGRF (in newtons and afterward normalized in body weight), of each subject: A total of 2,550 steps were analyzed. These variables were obtained for left and right feet; for seven movements, considering the ascending and descending phases of the movements, and global movement (includes both phases); at the cadences of 130 and $140 \mathrm{bpm}$; in both floor conditions. Version 13.0 of SPSS for Windows (Statistical Package for the Social Sciences, Chicago, IL) was used for statistical analysis: Mean and standard deviation values of all 15 subjects; Kolmogorov-Smirnov normality tests, and paired $t$ test, for comparison of the two conditions (platforms vs. step bench). Given the large number of paired $t$ tests performed $(42 \times$ $3)$, the Bonferroni correction was used in order to protect against Type I error. Thus, the significance level of 0.005 was adopted.

\section{Results}

Table 1 presents the mean and standard deviation values of 15 step-experienced subjects, of maximal and mean PP $\left(\mathrm{N} / \mathrm{cm}^{2}\right)$ and maximal VGRF (normalized in body weight), in left foot and in right foot; considering seven step movements performed at the cadences of 130 and $140 \mathrm{bpm}$ in two floor conditions. Table 2 presents the results for the ascending phase, and Table 3 presents the results for the descending phase of movements.

Table 1 Mean and SD of 15 Step-Experienced Subjects for Seven Step Movements, Considering Both Ascending and Descending Phases

\begin{tabular}{|c|c|c|c|c|c|c|}
\hline & \multicolumn{2}{|c|}{$\begin{array}{l}\text { Maximal plantar pressure } \\
\left(\mathrm{N} / \mathrm{cm}^{2}\right)\end{array}$} & \multicolumn{2}{|c|}{$\begin{array}{l}\text { Mean plantar pressure } \\
\left(\mathrm{N} / \mathrm{cm}^{2}\right)\end{array}$} & \multicolumn{2}{|c|}{$\begin{array}{c}\text { Maximal vertical } \\
\text { ground reaction force (BW) }\end{array}$} \\
\hline & Left foot & Right foot & Left foot & Right foot & Left foot & Right foot \\
\hline RB 130 pla & $32.87 \pm 9.32$ & $29.27 \pm 9.94$ & $11.90 \pm 1.70$ & $11.09 \pm 1.62$ & $1.43 \pm 0.14$ & $1.46 \pm 0.17$ \\
\hline RB 130 ben & $32.40 \pm 10.60$ & $28.20 \pm 9.32$ & $11.28 \pm 1.43$ & $10.71 \pm 1.54$ & $1.38 \pm 0.14$ & $1.45 \pm 0.21$ \\
\hline LB 130 pla & $31.40 \pm 8.46$ & $31.47 \pm 9.34$ & $11.29 \pm 1.35$ & $11.40 \pm 1.56$ & $1.63 \pm 0.29$ & $1.48 \pm 0.36$ \\
\hline LB 130 ben & $30.53 \pm 8.53$ & $30.20 \pm 8.97$ & $10.93 \pm 1.19$ & $11.33 \pm 1.81$ & $1.58 \pm 0.35$ & $1.45 \pm 0.40$ \\
\hline RR 130 pla & $42.67 \pm 11.00$ & $39.20 \pm 10.04$ & $14.00 \pm 2.17$ & $12.96 \pm 2.02$ & $2.30 \pm 0.23$ & $1.88 \pm 0.28$ \\
\hline RR 130 ben & $40.93 \pm 10.63$ & $38.40 \pm 13.87$ & $13.69 \pm 1.43$ & $12.08 \pm 1.56$ & $2.28 \pm 0.18$ & $1.91 \pm 0.21$ \\
\hline LR 130 pla & $34.80 \pm 8.56$ & $41.87 \pm 11.07$ & $12.64 \pm 1.60$ & $13.49 \pm 1.49$ & $1.90 \pm 0.25$ & $2.16 \pm 0.27$ \\
\hline LR 130 ben & $37.93 \pm 12.56$ & $39.80 \pm 13.43$ & $12.70 \pm 1.57$ & $13.54 \pm 1.88$ & $1.96 \pm 0.17$ & $2.23 \pm 0.23$ \\
\hline KL 130 pla & $34.27 \pm 11.04$ & $36.47 \pm 11.08$ & $12.40 \pm 1.67$ & $12.32 \pm 1.94$ & $1.50 \pm 0.14$ & $1.50 \pm 0.26$ \\
\hline KL 130 ben & $34.60 \pm 11.30$ & $34.33 \pm 9.68$ & $12.11 \pm 1.62$ & $11.96 \pm 1.87$ & $1.51 \pm 0.16$ & $1.49 \pm 0.18$ \\
\hline KH 130 pla & $36.93 \pm 10.83$ & $40.53 \pm 11.41$ & $13.19 \pm 1.83$ & $13.03 \pm 1.72$ & $1.77 \pm 0.21$ & $1.73 \pm 0.25$ \\
\hline KH 130 ben & $38.07 \pm 10.95$ & $38.33 \pm 12.25$ & $12.90 \pm 1.69$ & $12.63 \pm 1.70$ & $1.74 \pm 0.14$ & $1.74 \pm 0.18$ \\
\hline KR 130 pla & $41.13 \pm 11.67$ & $44.73 \pm 11.68$ & $14.14 \pm 1.82$ & $14.25 \pm 2.00$ & $1.70 \pm 0.23$ & $1.74 \pm 0.33$ \\
\hline KR 130 ben & $39.80 \pm 12.34$ & $40.40 \pm 12.94$ & $13.59 \pm 1.76$ & $13.26 \pm 2.10$ & $1.60 \pm 0.20$ & $1.59 \pm 0.24$ \\
\hline RB 140 pla & $33.20 \pm 10.07$ & $33.00 \pm 9.31$ & $11.80 \pm 1.69$ & $11.51 \pm 1.65$ & $1.50 \pm 0.16$ & $1.57 \pm 0.23$ \\
\hline RB 140 ben & $35.60 \pm 9.65$ & $31.07 \pm 9.90$ & $11.92 \pm 1.70$ & $11.29 \pm 1.67$ & $1.42 \pm 0.18$ & $1.53 \pm 0.21$ \\
\hline LB 140 pla & $33.87 \pm 10.94$ & $34.93 \pm 10.02$ & $11.50 \pm 1.38$ & $11.91 \pm 1.86$ & $1.71 \pm 0.27$ & $1.59 \pm 0.45$ \\
\hline LB 140 ben & $34.73 \pm 11.56$ & $34.27 \pm 10.86$ & $11.43 \pm 1.57$ & $11.75 \pm 1.99$ & $1.64 \pm 0.41$ & $1.56 \pm 0.46$ \\
\hline RR 140 pla & $43.13 \pm 12.39$ & $41.60 \pm 15.94$ & $14.05 \pm 2.23$ & $12.64 \pm 1.97$ & $2.41 \pm 0.24$ & $1.95 \pm 0.32$ \\
\hline RR 140 ben & $41.73 \pm 11.32$ & $38.13 \pm 14.74$ & $14.22 \pm 1.77$ & $12.59 \pm 2.03$ & $2.36 \pm 0.19$ & $2.01 \pm 0.19$ \\
\hline LR 140 pla & $38.47 \pm 12.51$ & $42.53 \pm 11.07$ & $12.90 \pm 1.74$ & $14.13 \pm 2.42$ & $1.99 \pm 0.23$ & $2.39 \pm 0.34$ \\
\hline LR 140 ben & $38.00 \pm 11.37$ & $38.53 \pm 14.04$ & $13.01 \pm 1.97$ & $13.68 \pm 2.01$ & $1.98 \pm 0.18$ & $2.34 \pm 0.24$ \\
\hline KL 140 pla & $34.80 \pm 11.83$ & $39.80 \pm 12.17$ & $12.75 \pm 1.81$ & $12.53 \pm 2.01$ & $1.58 \pm 0.16$ & $1.54 \pm 0.27$ \\
\hline KL 140 ben & $37.47 \pm 13.61$ & $35.93 \pm 11.91$ & $12.40 \pm 1.97$ & $12.24 \pm 2.01$ & $1.53 \pm 0.24$ & $1.56 \pm 0.23$ \\
\hline KH 140 pla & $37.07 \pm 10.88$ & $40.33 \pm 11.33$ & $12.87 \pm 1.74$ & $13.01 \pm 2.07$ & $1.73 \pm 0.18$ & $1.75 \pm 0.34$ \\
\hline KH 140 ben & $39.00 \pm 12.55$ & $39.20 \pm 13.78$ & $13.16 \pm 1.70$ & $12.92 \pm 2.17$ & $1.76 \pm 0.17$ & $1.76 \pm 0.22$ \\
\hline KR 140 lab & $43.67 \pm 13.35$ & $47.07 \pm 12.88$ & $14.13 \pm 1.71$ & $14.32 \pm 2.06$ & $1.77 \pm 0.24$ & $1.78 \pm 0.29$ \\
\hline KR 140 ben & $42.67 \pm 12.93$ & $43.00 \pm 13.80$ & $14.10 \pm 1.81$ & $13.92 \pm 2.25$ & $1.72 \pm 0.25$ & $1.66 \pm 0.23$ \\
\hline
\end{tabular}

Note. $\mathbf{R B}=$ right-basic-step $; \mathbf{L B}=$ left-basic-step $; \mathbf{R R}=$ right-run-step $; \mathbf{L R}=$ left-run-step $; \mathbf{K L}=$ knee-lift-step $; \mathbf{K H}=$ knee-hop-step $; \mathbf{K R}=$ kneetriple-repeater; obtained using pressure insoles when performing the movements at the cadences of 130 and $140 \mathrm{bpm}$, over a step bench (ben), and over two metal force platforms (pla). Statistical significance of the comparison of conditions in boldface $(p \leq 0.005)$. 
Table 2 Mean and SD of 15 Step-Experienced Subjects for Seven Step Movements, Considering Ascending Phase (on)

\begin{tabular}{|c|c|c|c|c|c|c|}
\hline & \multicolumn{2}{|c|}{$\begin{array}{c}\text { Maximal plantar pressure } \\
\left(\mathrm{N} / \mathrm{cm}^{2}\right)\end{array}$} & \multicolumn{2}{|c|}{$\begin{array}{c}\text { Mean plantar pressure } \\
\left(\mathrm{N} / \mathrm{cm}^{2}\right)\end{array}$} & \multicolumn{2}{|c|}{$\begin{array}{c}\text { Maximal vertical } \\
\text { ground reaction force (BW) }\end{array}$} \\
\hline & Left foot & Right foot & Left foot & Right foot & Left foot & Right foot \\
\hline $\mathrm{RB}$ on 130 pla & $19.27 \pm 3.92$ & $16.80 \pm 3.65$ & $7.43 \pm 1.02$ & $6.94 \pm 1.12$ & $1.10 \pm 0.16$ & $0.97 \pm 0.16$ \\
\hline $\mathrm{RB}$ on 130 ben & $19.27 \pm 5.52$ & $16.53 \pm 4.45$ & $7.57 \pm 1.37$ & $7.25 \pm 1.58$ & $1.13 \pm 0.14$ & $1.07 \pm 0.22$ \\
\hline LB on 130 pla & $18.07 \pm 4.38$ & $19.20 \pm 3.10$ & $7.08 \pm 0.91$ & $7.13 \pm 1.10$ & $1.11 \pm 0.32$ & $1.15 \pm 0.23$ \\
\hline LB on 130 ben & $16.33 \pm 3.85$ & $18.93 \pm 3.53$ & $6.84 \pm 0.78$ & $6.92 \pm 1.11$ & $1.03 \pm 0.19$ & $1.14 \pm 0.15$ \\
\hline RR on 130 pla & $42.33 \pm 11.32$ & $37.67 \pm 10.77$ & $13.79 \pm 2.41$ & $11.79 \pm 1.85$ & $2.26 \pm 0.28$ & $1.70 \pm 0.25$ \\
\hline RR on 130 ben & $40.40 \pm 9.88$ & $35.40 \pm 14.35$ & $13.44 \pm 1.96$ & $11.01 \pm 1.47$ & $2.26 \pm 0.19$ & $1.70 \pm 0.27$ \\
\hline LR on 130 pla & $34.53 \pm 8.80$ & $41.93 \pm 11.00$ & $11.34 \pm 1.34$ & $12.97 \pm 1.76$ & $1.74 \pm 0.30$ & $2.18 \pm 0.29$ \\
\hline LR on 130 ben & $34.33 \pm 11.70$ & $39.80 \pm 13.43$ & $11.86 \pm 1.97$ & $13.01 \pm 1.75$ & $1.80 \pm 0.35$ & $2.26 \pm 0.22$ \\
\hline KL on 130 pla & $19.60 \pm 3.09$ & $21.40 \pm 4.55$ & $8.14 \pm 0.84$ & $8.22 \pm 1.00$ & $1.11 \pm 0.10$ & $1.12 \pm 0.19$ \\
\hline KL on 130 ben & $20.00 \pm 4.61$ & $18.67 \pm 1.76$ & $7.93 \pm 0.99$ & $7.79 \pm 1.06$ & $1.13 \pm 0.08$ & $1.12 \pm 0.13$ \\
\hline $\mathrm{KH}$ on 130 pla & $30.13 \pm 12.45$ & $31.47 \pm 10.93$ & $11.21 \pm 1.84$ & $11.04 \pm 1.72$ & $1.69 \pm 0.25$ & $1.69 \pm 0.26$ \\
\hline $\mathrm{KH}$ on 130 ben & $30.67 \pm 12.70$ & $29.60 \pm 11.27$ & $10.81 \pm 1.60$ & $10.76 \pm 1.57$ & $1.68 \pm 0.14$ & $1.70 \pm 0.17$ \\
\hline KR on 130 pla & $40.20 \pm 7.48$ & $43.00 \pm 8.86$ & $12.79 \pm 1.34$ & $12.59 \pm 1.54$ & $1.99 \pm 0.43$ & $1.68 \pm 0.50$ \\
\hline $\mathrm{KR}$ on 130 ben & $39.50 \pm 8.33$ & $35.14 \pm 11.43$ & $13.86 \pm 2.33$ & $11.44 \pm 1.97$ & $2.02 \pm 0.59$ & $1.64 \pm 0.47$ \\
\hline $\mathrm{RB}$ on 140 pla & $20.27 \pm 7.59$ & $19.40 \pm 8.21$ & $8.01 \pm 2.33$ & $8.01 \pm 2.47$ & $1.17 \pm 0.19$ & $1.10 \pm 0.33$ \\
\hline $\mathrm{RB}$ on 140 ben & $18.47 \pm 3.66$ & $15.93 \pm 2.25$ & $7.32 \pm 1.31$ & $7.16 \pm 1.06$ & $1.13 \pm 0.18$ & $1.10 \pm 0.17$ \\
\hline LB on 140 pla & $17.27 \pm 4.73$ & $18.47 \pm 4.07$ & $6.95 \pm 0.88$ & $7.14 \pm 1.14$ & $1.07 \pm 0.23$ & $1.18 \pm 0.15$ \\
\hline LB on 140 ben & $16.73 \pm 3.77$ & $18.80 \pm 3.43$ & $6.81 \pm 0.97$ & $6.98 \pm 1.07$ & $1.01 \pm 0.13$ & $1.19 \pm 0.17$ \\
\hline RR on 140 pla & $43.13 \pm 12.39$ & $37.80 \pm 15.17$ & $14.47 \pm 2.53$ & $11.87 \pm 2.08$ & $2.41 \pm 0.24$ & $1.79 \pm 0.31$ \\
\hline RR on 140 ben & $41.07 \pm 11.15$ & $36.67 \pm 15.02$ & $13.96 \pm 2.03$ & $11.54 \pm 1.89$ & $2.36 \pm 0.19$ & $1.74 \pm 0.23$ \\
\hline LR on 140 pla & $37.73 \pm 12.77$ & $42.40 \pm 11.19$ & $12.16 \pm 2.37$ & $13.59 \pm 2.03$ & $1.84 \pm 0.22$ & $2.43 \pm 0.37$ \\
\hline LR on 140 ben & $36.13 \pm 12.54$ & $38.67 \pm 14.00$ & $12.23 \pm 2.42$ & $13.13 \pm 1.65$ & $1.77 \pm 0.38$ & 2.350 .23 \\
\hline $\mathrm{KL}$ on $140 \mathrm{pla}$ & $19.67 \pm 4.29$ & $20.73 \pm 4.86$ & $8.45 \pm 1.23$ & $8.29 \pm 1.44$ & $1.18 \pm 0.15$ & $1.14 \pm 0.22$ \\
\hline KL on 140 ben & $19.20 \pm 4.04$ & $21.67 \pm 6.76$ & $8.00 \pm 0.94$ & $8.05 \pm 1.33$ & $1.11 \pm 0.11$ & $1.19 \pm 0.11$ \\
\hline $\mathrm{KH}$ on 140 pla & $30.13 \pm 11.98$ & $31.20 \pm 11.25$ & $10.72 \pm 1.83$ & $10.50 \pm 2.01$ & $1.63 \pm 0.20$ & $1.62 \pm 0.24$ \\
\hline $\mathrm{KH}$ on 140 ben & $27.93 \pm 8.68$ & $29.40 \pm 10.55$ & $10.68 \pm 1.61$ & $10.45 \pm 1.78$ & $1.65 \pm 0.14$ & $1.69 \pm 0.20$ \\
\hline KR on 140 pla & $44.43 \pm 11.82$ & $41.36 \pm 14.01$ & $14.92 \pm 2.62$ & $12.20 \pm 2.29$ & $1.97 \pm 0.41$ & $1.75 \pm 0.37$ \\
\hline KR on 140 ben & $39.07 \pm 10.06$ & $40.93 \pm 12.93$ & $13.35 \pm 2.17$ & $12.71 \pm 2.01$ & $1.90 \pm 0.60$ & $1.70 \pm 0.47$ \\
\hline
\end{tabular}

Note. $\mathrm{RB}=$ right-basic-step $; \mathrm{LB}=$ left-basic-step $; \mathrm{RR}=$ right-run-step $; \mathrm{LR}=$ left-run-step $; \mathrm{KL}=$ knee-lift-step $; \mathrm{KH}=$ knee-hop-step $; \mathrm{KR}=$ kneetriple-repeater; obtained using pressure insoles when performing the movements at the cadences of 130 and $140 \mathrm{bpm}$, over a step-bench (ben), and over a metal force platform (pla). Statistical significance of the comparison of conditions in boldface $(p \leq 0.005)$.

Maximal plantar pressure ranged from 28.20 \pm 9.32 to $47.07 \pm 12.88 \mathrm{~N} / \mathrm{cm}^{2}$ considering both ascending and descending phases of the movements; considering ascending phase, it ranged from 15.93 \pm 2.25 to $44.43 \pm 11.82 \mathrm{~N} / \mathrm{cm}^{2}$; and considering descending phase it ranged from $24.47 \pm 6.57$ to $48.71 \pm 15.70 \mathrm{~N} / \mathrm{cm}^{2}$. In all conditions except one (descending phase of knee-triple-repeater-130 bpm), the PP obtained with metal platforms were greater. However, of the 42 statistical analyses performed, significant differences $(p=0.003)$ were observed only in one condition: ascending phase of knee-triple-repeater-130 bpm. This difference was observed in right foot (dominant side). Greater PP values were found in right leg (dominant side) and in movements with propulsion (hop and run).

Mean PP ranged from $10.71 \pm 1.54$ to $14.32 \pm$ $2.06 \mathrm{~N} / \mathrm{cm}^{2}$ considering global movements; considering ascending phase, it ranged from $6.81 \pm 0.97$ to $14.92 \pm 2.62 \mathrm{~N} / \mathrm{cm}^{2}$; and considering descending phase, it ranged from $9.61 \pm 1.45$ to $14.86 \pm 4.25$
$\mathrm{N} / \mathrm{cm}^{2}$. In all conditions except one (ascending phase of knee-triple-repeater-140 bpm), the PP obtained with metal platforms were greater. However, of the 42 statistical analyses performed, significant differences were observed only in three conditions: in knee-triple-repeater-130 bpm $(p=0.002)$; in descending phase of knee-triple-repeater-140 bpm ( $p=0.003)$; and in descending phase of knee-liftstep $140 \mathrm{bpm}(p=0.005)$. These differences were observed in right foot (dominant side). Mean PP values are greater in the nondominant side and in propulsion legs.

Maximal VGRF ranged from $1.38 \pm 0.14$ to $2.41 \pm 0.24 \mathrm{BW}$, considering global movements; considering ascending phase, it ranged from $0.97 \pm$ 0.16 to $2.43 \pm 0.37 \mathrm{BW}$; and considering descending phase, it ranged from $1.41 \pm 0.28$ to $2.06 \pm 0.33 \mathrm{BW}$. Of the 42 statistical analyses done, no significant differences $(p<0.005)$ were observed between both floor conditions. 
Table 3 Mean and SD of 15 Step-Experienced Subjects for Seven Step Movements, Considering Descending Phase (off)

\begin{tabular}{|c|c|c|c|c|c|c|}
\hline & \multicolumn{2}{|c|}{$\begin{array}{c}\text { Maximal plantar pressure } \\
\left(\mathrm{N} / \mathrm{cm}^{2}\right)\end{array}$} & \multicolumn{2}{|c|}{$\begin{array}{l}\text { Mean plantar pressure } \\
\left(\mathrm{N} / \mathrm{cm}^{2}\right)\end{array}$} & \multicolumn{2}{|c|}{$\begin{array}{c}\text { Maximal vertical } \\
\text { ground reaction force (BW) }\end{array}$} \\
\hline & Left foot & Right foot & Left foot & Right foot & Left foot & Right foot \\
\hline RB off 130 pla & $31.07 \pm 10.36$ & $29.20 \pm 9.95$ & $10.79 \pm 1.41$ & $10.62 \pm 1.34$ & $1.43 \pm 0.14$ & $1.46 \pm 0.17$ \\
\hline $\mathrm{RB}$ off 130 ben & $31.93 \pm 11.30$ & $28.00 \pm 9.63$ & $10.36 \pm 2.19$ & $9.82 \pm 1.57$ & $1.36 \pm 0.20$ & $1.41 \pm 0.28$ \\
\hline LB off 130 pla & $31.40 \pm 8.46$ & $31.47 \pm 9.34$ & $10.60 \pm 1.14$ & $10.34 \pm 1.05$ & $1.69 \pm 0.39$ & $1.53 \pm 0.42$ \\
\hline LB off 130 ben & $30.53 \pm 8.53$ & $30.13 \pm 9.05$ & $10.45 \pm 1.27$ & $9.79 \pm 1.59$ & $1.56 \pm 0.29$ & $1.42 \pm 0.29$ \\
\hline RR off 130 pla & $28.60 \pm 12.10$ & $33.40 \pm 10.87$ & $11.04 \pm 3.08$ & $11.32 \pm 1.79$ & $1.75 \pm 0.31$ & $1.82 \pm 0.35$ \\
\hline RR off 130 ben & $30.20 \pm 11.45$ & $32.67 \pm 11.31$ & $10.31 \pm 1.75$ & $10.92 \pm 1.30$ & $1.75 \pm 0.30$ & $1.88 \pm 0.20$ \\
\hline LR off 130 pla & $27.93 \pm 5.92$ & $24.47 \pm 6.57$ & $10.85 \pm 1.69$ & $9.61 \pm 1.45$ & $1.93 \pm 0.41$ & $1.65 \pm 0.30$ \\
\hline LR off 130 ben & $34.20 \pm 11.50$ & $27.07 \pm 11.64$ & $11.53 \pm 1.66$ & $10.31 \pm 1.89$ & $2.01 \pm 0.31$ & $1.83 \pm 0.30$ \\
\hline KL off $130 \mathrm{pla}$ & $34.27 \pm 11.04$ & $36.47 \pm 11.08$ & $11.45 \pm 1.52$ & $11.27 \pm 1.60$ & $1.50 \pm 0.14$ & $1.50 \pm 0.26$ \\
\hline KL off 130 ben & $34.60 \pm 11.30$ & $34.33 \pm 9.68$ & $11.29 \pm 1.61$ & $10.89 \pm 1.59$ & $1.51 \pm 0.16$ & $1.49 \pm 0.18$ \\
\hline $\mathrm{KH}$ off $130 \mathrm{pla}$ & $35.93 \pm 9.67$ & $40.20 \pm 11.77$ & $12.22 \pm 2.04$ & $11.73 \pm 1.40$ & $1.64 \pm 0.20$ & $1.61 \pm 0.26$ \\
\hline $\mathrm{KH}$ off 130 ben & $36.60 \pm 11.18$ & $37.20 \pm 12.29$ & $12.11 \pm 1.67$ & $11.45 \pm 1.34$ & $1.58 \pm 0.18$ & $1.55 \pm 0.22$ \\
\hline KR off 130 pla & $41.07 \pm 11.90$ & $31.20 \pm 8.44$ & $14.86 \pm 4.25$ & $10.98 \pm 1.41$ & $1.64 \pm 0.39$ & $1.66 \pm 0.36$ \\
\hline $\mathrm{KR}$ off 130 ben & $38.07 \pm 8.51$ & $40.64 \pm 10.60$ & $11.93 \pm 1.16$ & $11.12 \pm 1.46$ & $1.61 \pm 0.39$ & $1.67 \pm 0.44$ \\
\hline $\mathrm{RB}$ off 140 pla & $33.20 \pm 10.07$ & $33.00 \pm 9.31$ & $11.51 \pm 1.86$ & $10.87 \pm 1.28$ & $1.50 \pm 0.16$ & $1.57 \pm 0.23$ \\
\hline $\mathrm{RB}$ off 140 ben & $35.60 \pm 9.65$ & $31.07 \pm 9.90$ & $10.99 \pm 1.89$ & $10.42 \pm 1.40$ & $1.42 \pm 0.18$ & $1.53 \pm 0.21$ \\
\hline LB off 140 pla & $33.93 \pm 10.87$ & $35.20 \pm 9.76$ & $10.97 \pm 1.29$ & $10.80 \pm 1.41$ & $1.67 \pm 0.30$ & $1.60 \pm 0.38$ \\
\hline LB off 140 ben & $34.73 \pm 11.56$ & $34.13 \pm 11.01$ & $11.22 \pm 1.86$ & $10.43 \pm 1.68$ & $1.58 \pm 0.30$ & $1.49 \pm 0.28$ \\
\hline RR off 140 pla & $26.67 \pm 8.74$ & $35.13 \pm 14.59$ & $9.78 \pm 1.37$ & $11.23 \pm 1.74$ & $1.64 \pm 0.17$ & $1.92 \pm 0.31$ \\
\hline RR off 140 ben & $25.93 \pm 8.92$ & $30.87 \pm 8.38$ & $10.10 \pm 1.55$ & $11.07 \pm 1.75$ & $1.67 \pm 0.17$ & $2.00 \pm 0.20$ \\
\hline LR off 140 pla & $32.80 \pm 11.85$ & $28.13 \pm 9.34$ & $11.68 \pm 1.93$ & $10.45 \pm 1.82$ & $2.06 \pm 0.33$ & $1.81 \pm 0.46$ \\
\hline LR off 140 ben & $31.53 \pm 8.59$ & $25.00 \pm 8.87$ & $11.27 \pm 1.77$ & $9.85 \pm 1.57$ & $2.01 \pm 0.27$ & $1.82 \pm 0.28$ \\
\hline KL off 140 pla & $34.80 \pm 11.83$ & $39.80 \pm 12.17$ & $12.00 \pm 2.05$ & $11.69 \pm 1.75$ & $1.58 \pm 0.16$ & $1.54 \pm 0.27$ \\
\hline KL off 140 ben & $37.47 \pm 13.61$ & $35.93 \pm 11.91$ & $11.97 \pm 2.20$ & $11.05 \pm 1.55$ & $1.53 \pm 0.24$ & $1.56 \pm 0.23$ \\
\hline $\mathrm{KH}$ off 140 pla & $36.80 \pm 10.86$ & $40.00 \pm 11.10$ & $12.16 \pm 1.65$ & $11.97 \pm 1.57$ & $1.62 \pm 0.18$ & $1.66 \pm 0.32$ \\
\hline $\mathrm{KH}$ off 140 ben & $38.47 \pm 12.45$ & $36.93 \pm 13.20$ & $12.18 \pm 1.60$ & $11.51 \pm 1.68$ & $1.62 \pm 0.24$ & $1.60 \pm 0.26$ \\
\hline KR off 140 pla & $38.50 \pm 9.92$ & $48.71 \pm 15.70$ & $12.51 \pm 1.67$ & $12.65 \pm 1.99$ & $1.67 \pm 0.43$ & $1.72 \pm 0.40$ \\
\hline $\mathrm{KR}$ off 140 ben & $39.33 \pm 10.22$ & $38.80 \pm 11.85$ & $12.42 \pm 2.07$ & $11.64 \pm 1.56$ & $1.70 \pm 0.38$ & $1.69 \pm 0.40$ \\
\hline
\end{tabular}

\section{Discussion}

Ground reaction force is an essential variable to use in biomechanical modeling, usually obtained in laboratory using force platforms. A force platform surface is usually constructed of metal and this material is substantially different from materials used in step benches. It was hypothesized that mean and maximal PP and maximal VGRF obtained with pressure insoles would be significantly different when step movements are performed over metal platforms and over a step bench. In general, the PP obtained with metal platforms were greater, as well as in propulsion legs. However, very few statistical differences were observed due to floor conditions. The knee-triple-repeater was the task that presented more differences among conditions.

We must stress the fact that the sample rate of $50 \mathrm{~Hz}$ of the pressure insoles is too slow to measure "impact." However, our results suggest that PP obtained with metal platforms is close to those obtained step benches. No significant differences were obtained in most of the comparisons between the two conditions tested, when seven step movements were performed by 15 step-experienced subjects, leading us to conclude about ecological validity of the laboratory reproducibility of the conditions for the assessment of biomechanical parameters of step exercise. These results do not support our initial hypothesis. That the forces applied to the foot are similar with the softer step bench and the hard force platform surface is surprising but might reflect the ability of the performers to adapt their movement patterns in order to normalize the impact forces. Nevertheless, these findings need to be tested in inexperienced and older subjects.

Our results also suggest that due to the differences observed in right and left feet, this physical activity is not symmetric concerning mechanical load, leading us to take special care in choosing exercises to be performed by people of differing fitness status. Also, owing to differences observed among conditions of cadence and movement pattern, further research is needed concerning biomechanical analysis of recreational activity. 
Our results suggest that the conditions of practice of step exercise can be reproduced in laboratory in order to assess biomechanical loading variables. However, the present results are based on a sample of 15 female step instructors, physically active and with a long experience in this activity. It appears that professionals are able to provide musculoskeletal shock absorption without significantly increasing the muscle activation when performing on a rigid surface, such as in the study of Dowling and Benoit (1997). Thus, the force characteristics of the tasks may be different if participants with less experience in step are used. In conclusion, our finding is particularly important for the subsequent studies on biomechanical modeling, on which two metal force platforms have to be use, substituting the real conditions of practice, in order to collect GRF.

\section{Acknowledgments}

The authors wish to thank all the participants in this study. The authors wish to thank Maria de Lourdes Machado, Helô Isa André, and Pedro Mira (Faculty of Human Movement) for their help in data collection, and to Margarida Espanha and Paulo Armadada-Silva (Faculty of Human Movement) for their contributions to this manuscript.

\section{References}

Bartlett, R. (1999). Sports biomechanics: Reducing injury and improving performance. London: Spon Press.

Dixon, S.J., Collop, A.C., \& Batt, M.E. (2000). Surface effects on ground reaction forces and lower extremity kinematics in running. Medicine \& Science in Sports \& Exercise, 32, 1919-1926.

Dowling, J., \& Benoit, L. (1997). Biomechanical testing of a compliant bench for step aerobics. In Wilkerson, J.D., Zimmermann, W.J., \& Ludwig, K. (Eds.), Proceedings of the XV international symposium on biomechanics in sports (p. 149). Denton, TX: Human Texas University Press.

Dyson, R., \& Janaway, L. (2002). Effect of force platform surface on ground reaction peak force. In Hong, Y. (Ed.), International Research in Sports Biomechanics: ISBS (pp. 236-240). New York: Routledge.
Farrington, T., \& Dyson, R. (1995). Ground reaction forces during step aerobics. Journal of Human Movement Studies, 29, 89-98.

Hughes, J., Pratt, L., Linge, K., et al. (1991). Reliability of pressure measurements: the EMED F system. Clinical Biomechanics, 6, 14-18.

Maybury, M.C., \& Waterfield, J. (1997). An investigation into the relationship between step height and ground reaction forces in step exercise: A pilot study. British Journal of Sports Medicine, 31, 109-113.

Nigg, B.M., \& Herzog, W. (1999). Biomechanics of musculoskeletal system (2nd ed). Chichester: John Wiley \& Sons, Ltd.

Reebok University Press (1994). Introduction to step Reebok. Stoughton, MA: Reebok International, Ltd.

Ribeiro, J.K., \& Mota, C.B. (2004). Comportamento da força de reacção do solo durante a realização da marcha na ginástica de academia. Revista Brasileira de Biomecânica, 5(8), 49-55.

Salem, G.J., Ward, S.R., \& Lee, T.Q. (2000). Regulation of plantar-foot kinetics during exercises on step benches with markedly different structural properties. Journal of Strength and Conditioning Research, 14, 26-31.

Santos, D., Carline T., Flynn, L., Pitman, D., Feeney, D., Patterson, C., \& Westland, E. (2001). Distribution of in-shoe dynamic plantar foot pressures in professional football players. Foot, 11, 10-14.

Santos-Rocha, R., Veloso, A., Santos, H., Franco, S., \& PezaratCorreia, P. (2002). Ground reaction forces of step exercise depending on step frequency and bench height (pp. 156158). Proceedings of the XXth International Symposium on Biomechanics in Sports: International Society of Biomechanics in Sports, Cáceres, Spain,.

Scharff-Olson, M., Williford, H.N., Blessing, D.L., Moses, R., \& Wang, T. (1997). Vertical impact forces during benchstep aerobics: Exercise rate and experience. Perceptual and Motor Skills, 84, 267-274.

Williams, K.R. (2000). The Dynamics of running. In Zatsiorsky, V. (Ed.), Biomechanics in Sport: Performance enhancement and injury prevention (pp. 161-183). Oxford: Blackwell Science.

Winter, D.A. (2004). Biomechanics and motor control of human movement (3rd ed). New York: John Wiley \& Sons, Ltd. 